\title{
Immunosuppression of experimental autoimmune myasthenia gravis by mycophenolate mofetil
}

\author{
Sofie P.M. Janssen ${ }^{\text {a, } 1}$, Marko Phernambucq ${ }^{\text {a, }}$, Pilar Martinez-Martinez ${ }^{\text {a }}$, \\ Marc H. De Baets ${ }^{\mathrm{a}, \mathrm{b}}$, Mario Losen ${ }^{\mathrm{a}, *}$ \\ ${ }^{a}$ Department of Neuroscience, School of Mental Health and Neuroscience, Faculty of Health, Medicine and Life Sciences, Maastricht University, \\ Universiteitssingel 50, P.O. Box 616, 6200 MD Maastricht, The Netherlands \\ ${ }^{\mathrm{b}}$ Neuroimmunology Group, Biomedical Research Institute (BIOMED), Hasselt University, Diepenbeek, Belgium
}

Received 20 March 2008; received in revised form 13 May 2008; accepted 13 May 2008

\begin{abstract}
Currently used non-specific immunosuppressive drugs often require intervention in myasthenia gravis (MG) and clinical improvement varies widely. To analyze the therapeutic effect of mycophenolate mofetil (MMF) in experimental autoimmune MG (EAMG), rats were immunized with acetylcholine receptors (AChRs) and subsequently treated with MMF or vehicle. MMF treatment resulted in a significant suppression of anti-rat AChR antibody titers. Interestingly, no abnormalities of neuromuscular transmission and adverse side effects were detected in MMF-treated EAMG animals. Moreover, anti-rat AChR antibody titers correlated to an improvement of clinical outcome. In conclusion, our data suggest that MMF acts as a potent immunosuppressant drug in EAMG.
\end{abstract}

(C) 2008 Elsevier B.V. All rights reserved.

Keywords: Myasthenia gravis; Mycophenolate mofetil; Immunosuppression; Acetylcholine receptor; Neuromuscular junction

\section{Introduction}

Myasthenia gravis (MG) is a potentially life-threatening but treatable organ specific autoimmune disorder, characterized by weakness and fatigability of voluntary muscles, i.e. ocular, bulbar, limb and respiratory muscles. In about $85 \%$ of patients (Lindstrom et al., 1976), auto-antibodies against the nicotinic acetylcholine receptor (AChR) in the postsynaptic membrane of the neuromuscular junction are responsible for these symptoms, and have been shown to destroy neuromuscular transmission by different mechanisms: cross-linking of AChRs leading to increased AChR turnover, functional blockade of the acetylcholine-binding sites, complement-mediated damage to the postsynaptic membrane (Drachman, 1994), and destruction of proteins involved in neuromuscular formation (MartinezMartinez et al., 2007). Moreover, auto-antibodies that bind to

\footnotetext{
* Corresponding author. Tel.: +31 4338810 39; fax: +31 433671096 .

E-mail address:m.losen@np.unimaas.nl (M. Losen).

1 These authors contributed equally to this work.
}

the muscle specific protein kinase (MuSK), are present in a subgroup of patients with MG who do not have antibodies against the AChR (Hoch et al., 2001).

Currently many therapies are available for MG patients, all intervening with different targets of the disease. Thymectomy is nowadays a widely accepted and practiced form of treatment for MG and is performed in an attempt to induce complete remission or to reduce long-term need for immunosuppression (Gronseth and Barohn, 2000). In contrast, anti-cholinesterase agents are frequently used in the early disease course to decrease $\mathrm{MG}$ symptoms as they inhibit acetylcholine esterase from hydrolyzing acetylcholine. The prolonged exposure time of acetylcholine to AChRs in turn leads to improved neuromuscular transmission and muscle strength, however without affecting the autoimmune attack itself (Richman and Agius, 2003). Intravenous immunoglobulin (Gajdos et al., 2006) and plasmapheresis (Gajdos et al., 2006; Lehmann et al., 2006) are mostly temporary treatments used to produce rapid improvement during myasthenic crises or acute exacerbations. Finally, long-term immunosuppressives like corticosteroids, azathioprine, cyclosporine A, tacrolimus, 
rituximab, etanercept, leflunomide and cyclophosphamide are nowadays considered to be the most consistently effective and easily usable therapeutic agents as they induce improvement in approximately $80 \%$ of patients. However, the onset of clinical improvement varies widely among these immunosuppressive agents and side effects are often very severe due to toxicity of the therapeutics (Ciafaloni, 2005; Garcia-Carrasco et al., 2007; Sieb, 2005). Currently, the best evidence based immunosuppressive therapy for MG is the combination of prednisone with azathioprine. This combined treatment allows a reduction of required prednisone and concomitantly reduces, albeit incompletely, side effects such as "malaise, back pain, visual deterioration, and rash" (Palace et al., 1998). From this point of view, new effective and safe immunosuppressive drugs for $M G$ patients are urgently required.

Mycophenolate mofetil (MMF) is a potent immunosuppressive drug that was primarily licensed to prevent rejection of transplanted organs (European Mycophenolate Mofetil Cooperative Study Group, 1995) and then introduced for the therapy of autoimmune diseases of skin (Enk and Knop, 1997), gut (Neurath et al., 1999), and eye (Larkin and Lightman, 1999), in rheumatoid arthritis (Goldblum, 1993), lupus nephritis (Ginzler et al., 2005) and Wegener's granulomatosis (Nowack et al., 1999). In the last decennium it has also been used in patients with neuromuscular diseases like inflammatory myopathy and chronic inflammatory demyelinating polyradiculoneuropathy to achieve either symptom stabilization or remission (Chaudhry et al., 2001). MMF operates through reduction of intracellular purine synthesis in both B- and T-lymphocytes, leading to inhibition of lymphocyte proliferation. Moreover, MMF does not only augment apoptosis of lymphocytic and monocytic cell lines (Cohn et al., 1999) and reduces the levels of immunoglobulin isotypes $\mathrm{M}, \mathrm{G}$ and A produced by polyclonal activated Blymphocytes (Eugui et al., 1991), it also inhibits glycosylation of adhesion molecules (Blaheta et al., 1998) and regulates the secretion of inflammatory and anti-inflammatory cytokines (Durez et al., 1999). Consequently, MMF acts as a pluripotent immunomodulator in numerous types of immunological diseases (Schneider-Gold et al., 2006).

The successful use of MMF in MG has already been described in several small clinical trials, with the main advantage of MMF being its tolerability and safety profile (Caponnetto et al., 2001; Chaudhry et al., 2001; Ciafaloni et al., 2001; Hauser et al., 1998; Lim et al., 2007; Meriggioli et al., 2003a; Meriggioli et al., 2003b; Mowzoon et al., 2001; Prakash et al., 2007; Schneider et al., 2001). However, data from 2 recently completed large phase 3 prospective, multicenter, placebo-controlled trials showed only little or no effect of the drug when used as an adjunctive treatment to corticosteroids (Hampton, 2007; Sanders et al., 2008; The Muscle Study Group, 2008). This suggests that further studies are needed to assess the benefits of MMF as primary or long-term therapy and therefore encourages the examination of its precise mechanism in an experimental animal model of MG.

Experimental autoimmune myasthenia gravis (EAMG) in rats induced by immunization with AChRs from the electric organ of Electrophorus electricus or Torpedo californica, is already known for decades to be a reproducible and characteristic chronic model of MG (De Baets, 2003). Indeed, the presence of antibodies directed to rat muscle AChRs in the circulation of rats with EAMG provides evidence for the existence of autoimmunity in this experimental disease model. Moreover, similar to MG, antigenic modulation and complement-mediated focal damage of the postsynaptic membrane are the main pathogenic mechanisms (Losen et al., 2008) that lead to muscular weakness, hunched posture, weight loss and electrophysiological abnormalities in these animals, and can be relieved by anti-cholinesterases (Lennon et al., 1975). For these reasons, EAMG in rats is an excellent model to examine the therapeutic effect of MMF.

\section{Materials and methods}

\subsection{Animal model}

Eight-week old female Lewis rats were obtained from the Department of Experimental Animal Services, University of Maastricht, The Netherlands, with permission of the Committee on Animal Welfare, according to Dutch governmental rules.

For immunization and intubation, rats were anesthetized by inhalation of $3-5 \%$ isoflurane in air enriched with $50 \%$ oxygen, supplied by a cylindrical cap held over the head. Euthanasia was performed by $\mathrm{CO}_{2} /$ air inhalation and subsequent cervical dislocation.

\subsection{Induction of chronic EAMG}

Animals were immunized at the base of the tail with $10 \mu \mathrm{g}$ T. californica AChR $(t \mathrm{AChR})$ in $0.1 \mathrm{~mL}$ of phosphate-buffered saline (PBS), emulsified in an equal amount of Freund's adjuvant with $0.1 \%$ Mycobacterium tuberculosis (Difco Laboratories, Detroit, USA) (Lennon et al., 1975). Blood samples were taken weekly from the vena saphena magna to measure anti-rat AChR antibody titers. Sham-immunization was performed under similar conditions using $0.1 \mathrm{~mL}$ of PBS and an equal volume of Freund's adjuvant with $0.1 \%$ M. tuberculosis (CFA) (Lennon et al., 1975).

\subsection{Administration of immunosuppressive drugs}

Mycophenolate mofetil (MMF; Roche Palo Alto LLC, California) was administered orally at a dose of $30 \mathrm{mg} / \mathrm{kg} /$ day in a $900 \mu \mathrm{L}$ autoclaved vehicle solution consisting of distilled water, $0.9 \%$ benzyl alcohol, $0.4 \%$ polysorbate $80,0.9 \%$ sodium chloride, $0.5 \%$ carboxymethylcellulose and $5.0 \%$ sucrose $(\mathrm{pH} 3.5)$.

\subsection{Experimental design}

Two different treatment regimes of MMF were investigated using 62 rats in total. For the first treatment regime, two groups of rats immunized with $t \mathrm{AChR}$ were given either vehicle (vehicle-treated EAMG rats, $n=10$ ) or MMF (MMF-treated EAMG rats, $n=10$ ) daily for 3 weeks, starting at the time of immunization, and were compared to sham-immunized controls 
$(n=10) 8$ weeks after immunization. For this series in particular, immunohistochemical analyses were performed together with determination of total muscle AChR content.

For the second treatment regime, two groups of rats immunized with $t \mathrm{AChR}$ received either vehicle (vehicle-treated EAMG rats, $n=18$ ) or MMF (MMF-treated EAMG rats, $n=10$ ) daily for 8 weeks, starting at the time of immunization. Four sham-immunized rats were used as controls and were compared to the above-mentioned groups 8 weeks after immunization. More precisely, electromyographic measurements (EMG) during continuously intravenous curare infusions to induce decrement of compound muscle action potentials (CMAP) were performed.

For all rats, the body weights, clinical scores and anti-rat $\mathrm{AChR}$ antibody titers were measured regularly.

\subsection{Clinical scoring}

The severity of clinical signs in EAMG was scored weekly by measuring rats' muscular weakness through assessing their ability to grasp and lift repeatedly a 300-g rack from the table while suspended manually by the base of the tail for $30 \mathrm{~s}$ (Hoedemaekers et al., 1997). Clinical scoring was based on the presence of tremor, hunched posture, muscle strength and fatigability. Disease severity was expressed as 0 , no obvious abnormalities; I, no abnormalities before testing but reduced strength at the end; II, clinical signs present before testing i.e. tremor, hunched posture, head down, weak grip; III, severe clinical signs present before testing, no grip, moribund (Lennon et al., 1975).

\subsection{Measurement of serum anti-rat AChR antibody titers}

Anti-rat AChR antibody titer measurements were carried out at $4{ }^{\circ} \mathrm{C}$ by a double-antibody radioimmunoassay (RIA) as described previously (Losen et al., 2005; Martinez-Martinez et al., 2007). Briefly, antibodies directed against rat AChRs were measured using a crude extract of denervated rat muscle (approximately $5 \mathrm{nmol} / \mathrm{L} \mathrm{AChR}$ ), labeled with an excess of ${ }^{125} \mathrm{I}$ $\alpha$-bungarotoxin (IM109, 6.12 TBq/mmol, GE Healthcare, Amersham Place, UK), and incubated overnight with $5 \mu \mathrm{L}$ rat test serum. Precipitation was induced by addition of $100 \mu \mathrm{L}$ goat anti-rat IgG serum, followed by 3 washing steps in PBS with $0.5 \%$ Triton X-100. Radioactivity counts were measured in a $\gamma$-counter (1480 Wizard 3", PerkinElmer, USA) and corrected for background measurements using normal rat serum. Antibody titers were expressed as nmoles of $\alpha$-bungarotoxin binding sites/L.

\subsection{Immunohistochemical staining}

Left tibialis anterior muscles were frozen in isopentane cooled with liquid nitrogen to perform immunohistochemical staining as described previously (Losen et al., 2005; MartinezMartinez et al., 2007) with the following modifications: $10 \mu \mathrm{m}$ cryosections were incubated with mouse anti-rapsyn mAb 1234 (1:500 in PBS with $2 \%$ bovine serum albumin (PBSA), Sigma, USA), rabbit anti-vesicular acetylcholine transporter (VAChT,
1:500 in PBSA, Sigma, USA), and Alexa 594-conjugated $\alpha$-bungarotoxin (1:300 in PBSA, Molecular Probes, USA) for $1 \mathrm{~h}$. Subsequently, sections were incubated for $45 \mathrm{~min}$ with the appropriate secondary antibodies: biotinylated goat antimouse IgG (1:400 in PBSA; Jackson Immunoresearch, USA) and Alexa 350-conjugated goat anti-rabbit (1:100 in PBSA; Molecular Probes, USA). Finally, the biotinylated secondary antibody was stained for 30 min with Alexa 488-conjugated streptavidin (1:2000 in PBSA; Molecular Probes, USA). All incubation steps were performed at room temperature.

\subsection{Immunofluorescence microscopy}

Pictures of triple stained muscle sections were taken using a Provis AX70 fluorescent microscope (Olympus, Hamburg, Germany) connected to a black and white digital video camera (U-CMAD-2, Olympus), equipped with AnalySIS software (Soft Imaging Systems, Münster, Germany). All microscope settings were maintained constant during photographing, with the exposure time of the camera set to values that prevent saturation. Pictures were subsequently analyzed using ImageJ software (version 1.37k; http://rsb.info.nih.gov/ij/). Neuromuscular junctions were identified as regions with presynaptic VAChT staining.

\subsection{Measurement of total muscle AChR}

The right tibialis anterior muscle of each rat was minced and homogenized with an Ultra-Turrax ( 3 times $30 \mathrm{~s}$ at $4{ }^{\circ} \mathrm{C}$ ) in $10 \mathrm{~mL}$ extraction buffer (PBS mixed with $10 \mathrm{mM}$ sodium azide, $10 \mathrm{mM}$ ethylenediaminetetraacetic acid, $10 \mathrm{mM}$ iodoacetamide, and $1 \mathrm{mM}$ phenylmethyl sulfonyl fluoride). The homogenate was centrifuged at $22,100 \mathrm{~g}$ for $30 \mathrm{~min}$ at $4{ }^{\circ} \mathrm{C}$ and the resulting pellet was resuspended in $2.5 \mathrm{~mL}$ extraction buffer with an additional 2\% Triton X-100. Extraction was performed on a reciprocal shaker for $1 \mathrm{~h}$ at $4{ }^{\circ} \mathrm{C}$, followed by centrifugation at $22,100 \mathrm{~g}$ for $30 \mathrm{~min}$ at $4{ }^{\circ} \mathrm{C}$. AChR concentrations were then measured by RIA using an excess of ${ }^{125} \mathrm{I}-\alpha$-bungarotoxin (IM209, 74 TBq/mmol, GE healthcare, Amersham Place, UK) as described previously (Losen et al., 2005). Total muscle membrane AChR concentration was calculated per $\mathrm{g}$ of fresh muscle and thus expressed in $\mathrm{fmol} / \mathrm{g}$.

\subsection{Electromyography}

Decrement of CMAP during repetitive nerve stimulation was measured during concurrently continuous curare (D-tubocurarine chloride, ICN Biomedicals, Illkirch France) infusions in left tibialis anterior muscles of rats 8 weeks after immunization using the EMG system Viking IV (Nicolet Biomedicals Inc., Madison, USA) as previously described (Losen et al., 2005; Martinez-Martinez et al., 2007; Seybold et al., 1976) with the following modifications: curare infusions (Terfusion syringe pump, model STC-521, Terumo, Japan; $1 \mathrm{~mL} / \mathrm{h}, 0.33 \mu \mathrm{g}$ curare/ $\mathrm{min}$ ) were proceeded after intubation and catheterization of the tail vene. To detect a decrementing response, series of 8 supramaximal stimuli were given to the nervus tibialis at $3 \mathrm{~Hz}$ 
with stimulus duration of $0.2 \mathrm{~ms}$ and an interval of $2 \mathrm{~min}$. The signal was considered positive when both amplitude and area of the fourth negative peak of the CMAP showed a decrease of at least $10 \%$ when compared to the first response (Losen et al., 2005; Martinez-Martinez et al., 2007). To demonstrate reproducibility, a minimum of three recordings were made with an interval of at least $30 \mathrm{~s}$ between the repetitive stimulations. During the measurements, skin temperature was kept between $35^{\circ} \mathrm{C}$ and $37^{\circ} \mathrm{C}$ by means of a heating pad.

\subsection{Statistics}

GraphPad Prism 4 version 4.00 was used to perform statistical analyses. Comparison between normally distributed values was performed using an unpaired $t$-test or one-way analysis of variance (ANOVA) in which differences between means were subsequently assessed using Tukey's multiple comparison post hoc testing. For not-normally distributed values, a Mann-Whitney test or Kruskall-Wallis test with Tukey's multiple post hoc comparison analysis was performed to assess differences between mean values. Correlations were expressed using Pearson's correlation coefficients and represented by non-linear regression using the $95 \%$ confidence interval. A two-sided probability value $<0.05$ was considered significant. Values are expressed as means \pm standard deviation unless stated otherwise.

\section{Results}

\subsection{MMF treatment suppresses the development of anti-rat AChR antibodies in EAMG}

As new effective and safe immunosuppressive drugs are urgently required to improve MG therapy, MMF was tested for its immunosuppressive action in an animal model of MG. In the present study, EAMG rats were treated for 3 or 8 weeks with MMF, starting from the day of immunization. Subsequently, antirat AChR antibody titers were measured weekly for 8 weeks to determine whether MMF may act immunosuppressive.

Data of both 3 and 8 weeks of MMF treatment are represented together since statistical analyses confirmed similar results in antibody titers during the whole time course. As expected, sham-immunized control rats did not develop anti-rat AChR antibodies. Anti-rat AChR antibody titers of vehicletreated EAMG rats started to develop 2 weeks after immunization, and continuously increased until the end of treatment $(9.0 \pm$ $7.0 \mathrm{nmol} / \mathrm{L}$; Fig. 1A). Interestingly, anti-rat $\mathrm{AChR}$ antibody titers of EAMG animals treated for 3 weeks with MMF remained fully suppressed up to 4 weeks after immunization. From week 5 on, anti-rat AChR antibodies increased slightly, reaching a maximum value of only $0.9 \pm 1.3 \mathrm{nmol} / \mathrm{L}$ at the end of the experimental period. A similar increase occurred also in rats that continued MMF treatment during the entire experimental period. The average antibody titer of MMF-treated EAMG animals was significantly different from vehicle-treated EAMG rats $(p<0.001$; Fig. 1B). Although, on the one hand $35 \%$ of MMF-treated EAMG rats still possessed antibody titers
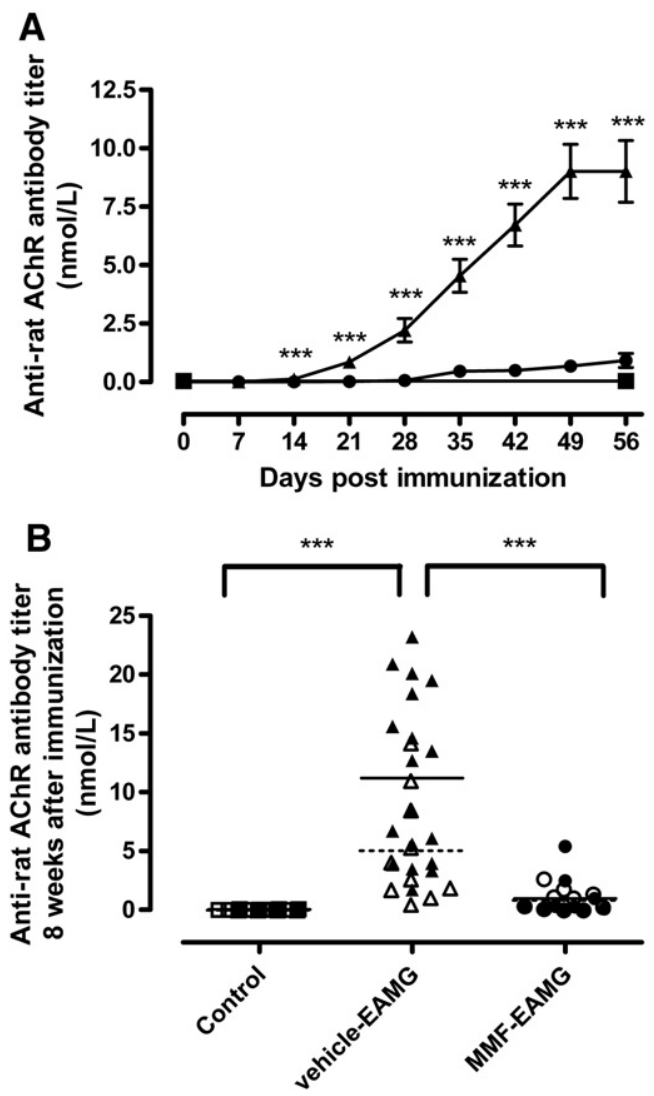

Fig. 1. Anti-rat AChR antibody titers in female Lewis rats, immunized at the age of 8 weeks (day 0 ) with $10 \mu \mathrm{g}$ of $t \mathrm{AChR}$ in CFA or with CFA only. Upper panel (A) represents the time course of average anti-rat AChR antibody titers of both 3 and 8 weeks MMF treatment regimes together up to 8 weeks after immunization. Control rats $(n=14)$ are represented by closed squares, vehicle-treated EAMG animals $(n=26)$ by closed triangles and MMF-treated EAMG rats $(n=20)$ by closed circles. Data are expressed as mean \pm standard error. Bottom panel (B) corresponds to individual anti-rat AChR antibody titers 8 weeks after immunization. Open symbols correspond to the 3 week MMF treatment and closed symbols to the 8 week MMF treatment. Dashed horizontal lines represent average values in the 3 weeks MMF administration group, while full horizontal lines represent average values after 8 weeks of MMF treatment. Note that MMF administration almost completely blocked average anti-rat AChR antibody titers (average endpoint value of $0.9 \pm 1.3 \mathrm{nmol} / \mathrm{L}$ ) when compared to vehicle-treated EAMG animals $(9.0 \pm 7.0 \mathrm{nmol} / \mathrm{L}, p<0.001)$. ANOVA $p$ values in upper panel and Tukey post hoc $p$ values in bottom panel; *** $p<0.001$.

between 1.0 and $5.4 \mathrm{nmol} / \mathrm{L}$, on the other hand $30 \%$ of MMFtreated EAMG rats did not develop detectable anti-rat $\mathrm{AChR}$ antibodies at all (Fig. 1B); unlike in the untreated EAMG group, where all animals developed detectable anti-AChR titers.

\subsection{Reduction of anti-rat AChR antibody titers improves clinical outcome in EAMG}

\subsubsection{Clinical assessment}

To examine the relation between low or high anti-rat AChR antibody titers and the incidence of MG symptoms, rats were scored weekly for clinical signs. At the time of immunization, body weight was similar for all groups of rats both in the 3 and 8 weeks MMF treatment study (average values of $145 \pm 7$ and $150 \pm 9$ g, respectively). Eight weeks later, body weight was 
equally increased in all animals, reaching average values up to $196 \pm 10$ and $204 \pm 9 \mathrm{~g}$ after 3 and 8 weeks of treatment, respectively (Fig. 2A). In the vehicle-treated EAMG group 6 animals developed mild to severe EAMG signs, 3 of which had to be euthanized in advance due to more than $15 \%$ of weight loss and very severe clinical signs (score III). In comparison, in the MMF-treated EAMG group, only one animal exerted moderate clinical EAMG signs (score II; Fig. 2B). In conclusion, MMF-induced suppression of anti-rat AChR antibody titers clearly decreased the severity of clinical signs. Importantly, in the MMF-treated animals no adverse side effects such as loss of body weight, fever, diarrhea, dehydration or breathing difficulties were observed.

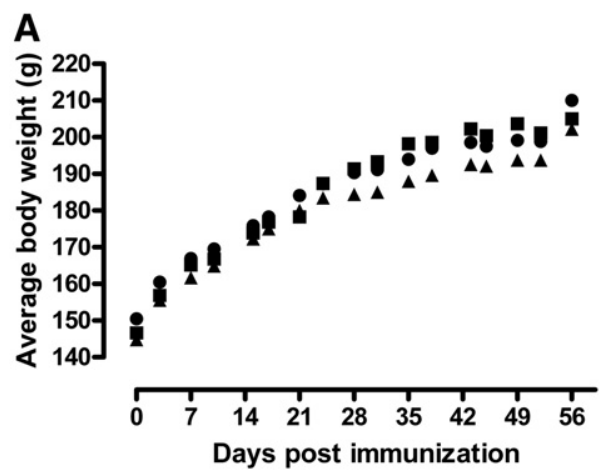

B

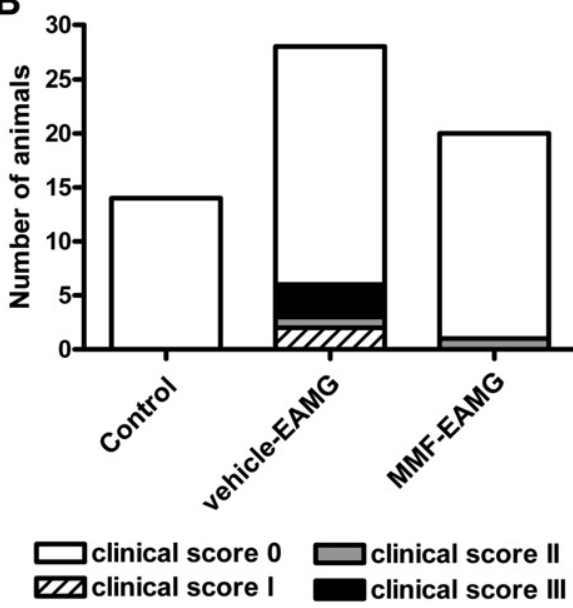

Fig. 2. Body weight and clinical myasthenia gravis score. Upper panel (A) represents the time course of body weight of all rats of both the 3 and 8 week treatment regimes together, starting from the day of immunization (day 0) up to 8 weeks after immunization. Control rats $(n=14)$ are represented by closed squares, vehicle-treated EAMG animals $(n=28)$ by closed triangles and MMFtreated EAMG rats $(n=20)$ by closed circles. Data are expressed as mean values. Note that there are no differences in gaining weight between the groups. Bottom panel (B) shows the clinical myasthenia gravis score 8 weeks after immunization of all rats of both MMF treatment regimes together (control: $n=14$; vehicleEAMG: $n=28$; MMF-EAMG: $n=20$ ). Open bars represent the number of clinically healthy animals (score 0 ), hatched bars represent the number of mildly sick animals (score I), grey bars represent the number of moderately ill animals (score II) and solid bars represent the number of severely ill animals (score III). Note that MMF treatment clearly decreased the appearance of clinical myasthenia gravis signs as only one MMF-treated EAMG animal with score II could be observed.

\subsubsection{Neuromuscular transmission and total muscle membrane AChR concentration}

The clinical efficiency of suppressed anti-rat AChR antibody titers was investigated by quantitative assessment of neuromuscular transmission in the left tibialis anterior muscle during intravenous curare infusion in rats treated for 8 weeks with MMF or vehicle. Curare is known to specifically bind the nicotinic AChR, thereby inhibiting normal functioning of the AChR ion channel. From this point of view, the amount of curare needed to observe decrement of the CMAP was used as a measurement for rat's clinical status.

Fig. 3A shows that MMF-treated EAMG rats exerted decrementing responses at curare doses in the range of shamimmunized control rats (average values of $8.8 \pm 1.9$ vs $11.6 \pm$ $1.2 \mu \mathrm{g}$, respectively), while decrement was observed at significantly lower curare doses in vehicle-treated EAMG animals $(5.8 \pm 2.1 \mu \mathrm{g} ; p<0.01$ vs MMF-EAMG; $p<0.001$ vs control). Interestingly, an exponential correlation was found between the anti-rat AChR antibody titers and the amount of intravenously infused curare in order to observe decrementing responses $\left(y=5.1 * \mathrm{e}^{-0.48 x}+5.3 ; p<0.001 ; R^{2}=0.60\right.$; Fig. $\left.3 \mathrm{~B}\right)$. In particular, it seemed that increases in anti-rat AChR antibody titers above $1.0 \mathrm{nmol} / \mathrm{L}$ already provoked decrementing responses at about $50 \%$ lower curare levels compared to healthy control animals. Since $88 \%$ of MMF-treated EAMG rats studied under this experimental condition could suppress antirat $\mathrm{AChR}$ antibody titers even below $1.0 \mathrm{nmol} / \mathrm{L}, \mathrm{MMF}$ efficiently improved neuromuscular transmission in EAMG.

To further analyze the effect of MMF immunosuppression on the neuromuscular junction, the AChR concentrations were determined in tibialis anterior muscles of rats treated for 3 weeks with MMF or vehicle. Surprisingly, average membrane AChR concentrations of vehicle-treated and MMFtreated EAMG rats $(9 \pm 3$ and $11 \pm 2 \mathrm{fmol} / \mathrm{g}$, respectively) were both significantly decreased by about $45 \%$ when compared to sham-immunized control rats $(18 \pm 3 \mathrm{fmol} / \mathrm{g}$; post hoc $p<0.001)$. However, several individual AChR concentration values of MMF-treated EAMG animals were in the range of those of sham-immunized controls, and showed thus no evidence of muscle membrane AChR breakdown. Similarly to the aforementioned curare resistance measurements of neuromuscular transmission, an exponential correlation was found between the anti-rat AChR antibody titers and the total muscle AChR concentration (Fig. 3C). Indeed, only a slightly increased anti-rat AChR antibody titer of about $1.0 \mathrm{nmol} / \mathrm{L}$ was already sufficient to reduce the normal amount of total membrane AChRs by half $\left(y=8.7 * \mathrm{e}^{-2.96 x}+8.8 ; p<0.001\right.$; $\left.R^{2}=0.68\right)$.

Fluorescent immunohistochemical studies on contralateral tibialis anterior muscles of the same rats treated for 3 weeks with MMF or vehicle confirmed the impact of different anti-rat AChR antibody titers on the postsynaptic membrane of the neuromuscular junction (Fig. 4). Indeed, compared to controls, triple fluorescent staining of neuromuscular endplates showed a clearly reduced postsynaptic AChR staining in almost all vehicle-treated EAMG rats while the AChR concentration in the neuromuscular junction of MMF-treated EAMG animals was 
variable. More precisely, in MMF-treated EAMG rats with antirat $\mathrm{AChR}$ antibody titers below about $0.5 \mathrm{nmol} / \mathrm{L}$, almost all neuromuscular junctions stained intensely for AChRs, comparable to those of control rats, while most AChRs seemed to be broken down in rats expressing higher anti-rat AChR antibody titers. Similar findings were observed for the staining intensity of the receptor associated protein rapsyn. As expected, presynaptic VAChT staining did not differ between the groups. In conclusion for these biochemical and histochemical findings, it seemed that MMF could prevent breakdown of total muscle membrane AChRs as a result of decreased anti-rat $\mathrm{AChR}$ antibody titers.
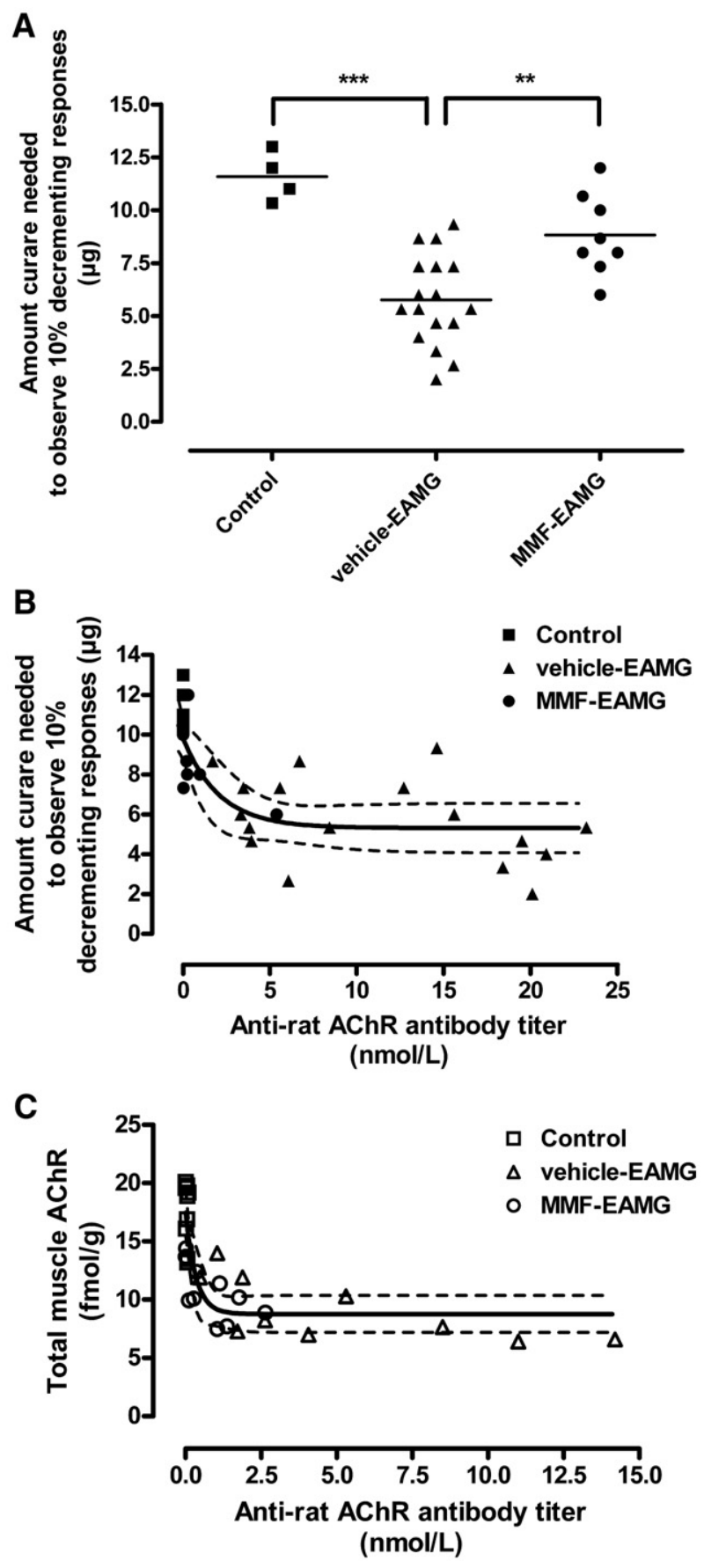

\section{Discussion}

Auto-antibodies directed against the $\alpha$-subunit of the AChR on the postsynaptic membrane of the neuromuscular junction are thought to be the main cause of neuromuscular transmission failure in $\mathrm{MG}$, resulting in skeletal muscle fatigue and weakness (De Baets and Stassen, 2002). From this point of view, one of the therapeutic approaches often used for autoimmune MG includes immunosuppressive therapy. Unfortunately, all currently used immunosuppressive drugs carry serious side effects and are not tolerated or do not cause an adequate response in some patients (Ciafaloni, 2005; Garcia-Carrasco et al., 2007; Sieb, 2005). Therefore, the possibility of a new effective, safe and specific immunosuppressive agent to add to the list of $\mathrm{MG}$ treatments is very attractive (Ciafaloni, 2005).

The successful use of MMF in MG was first described a decade ago in a girl who had previously failed treatment with azathioprine, prednisone and cyclosporine (Hauser et al., 1998). This case report subsequently prompted investigators to study the effects of MMF in more detail in MG. Adverse events are thought to be absent during treatment, as MMF only interferes with proliferating antibody producing B- and T-lymphocytes, due to inhibition of inosine monophosphate dehydrogenase type II (IMPDH II), a key enzyme in the de novo pathway of purine synthesis (Allison and Eugui, 2000; Schneider-Gold et al., 2006). Furthermore, as IMPDH II is not involved in the hypoxantine guanine phosphoribosyl transferase salvage pathway of purine synthesis, MMF does not inhibit key enzymes of other cell tissues, which is different from other immunosuppressants that are nowadays used for treatment of MG patients (Patel et al., 2006; Schneider-Gold et al., 2006). For example,

Fig. 3. Clinical and biochemical characteristics of the tibialis anterior muscle 8 weeks after immunization in controls, vehicle- and MMF-treated EAMG animals. Upper panel (A) represents the detection of $10 \%$ decrementing responses during intravenous curare infusions $(1 \mathrm{~mL} / \mathrm{h} ; 0.33 \mu \mathrm{g}$ curare $/ \mathrm{min})$, while middle and bottom panel represent the exponential correlation between anti-rat AChR antibody titers and clinical characteristics (B) or total muscle AChR levels $(\mathrm{C})$, respectively. In panels $\mathrm{A}$ and $\mathrm{B}$, which represent data of 8 weeks of MMF treatment regime, sham-immunized controls $(n=4)$ are represented by closed squares, vehicle-treated EAMG animals $(n=17)$ by closed triangles and MMF-treated EAMG rats $(n=8)$ by closed circles. In contrast, in panel C, which represents data of 3 weeks of MMF treatment regime, shamimmunized controls $(n=10)$ are represented by open squares, vehicle-treated EAMG animals $(n=10)$ by open triangles and MMF-treated EAMG rats $(n=10)$ by open circles. Mean values are indicated by horizontal lines in all panels, while dashed lines represent the $95 \%$ confidence interval in panels B and C. Note the significant decrease in the amount of intravenously infused curare in order to detect decrementing responses in vehicle-treated EAMG animals $(5.8 \pm 2.1 \mu \mathrm{g})$ when compared to controls $(11.6 \pm 1.2 \mu \mathrm{g}, p<0.001)$. Interestingly, there was a significant increase in the amount of intravenously infused curare when EAMG rats were treated with MMF $(8.8 \pm 1.9 \mu \mathrm{g}, p<0.01$; panel A). Moreover, an exponential correlation could be observed between the amount of intravenously infused curare and anti-rat AChR antibody titers $\left(y=5.1 * \mathrm{e}^{-0.48 x}+5.3\right.$; $p<0.001 ; R^{2}=0.60$; B) 8 weeks after immunization. Note that anti-rat AChR antibody titers above $1.0 \mathrm{nmol} / \mathrm{L}$ already caused decrementing responses at about $50 \%$ of normal curare values. Correlation analysis between total tibialis anterior AChR concentration and anti-rat AChR antibody titers $\left(y=8.7 * \mathrm{e}^{-2.96 x}+8.8 ; \quad p<0.001 ; R^{2}=0.68 ;\right.$ panel $\left.\mathrm{C}\right)$ further revealed that antibody titers above $1.0 \mathrm{nmol} / \mathrm{L}$ provoked a $50 \%$ decrease in total muscle AChR concentration. Tukey post hoc ${ }^{* *} p<0.01,{ }^{* * *} p<0.001$. 

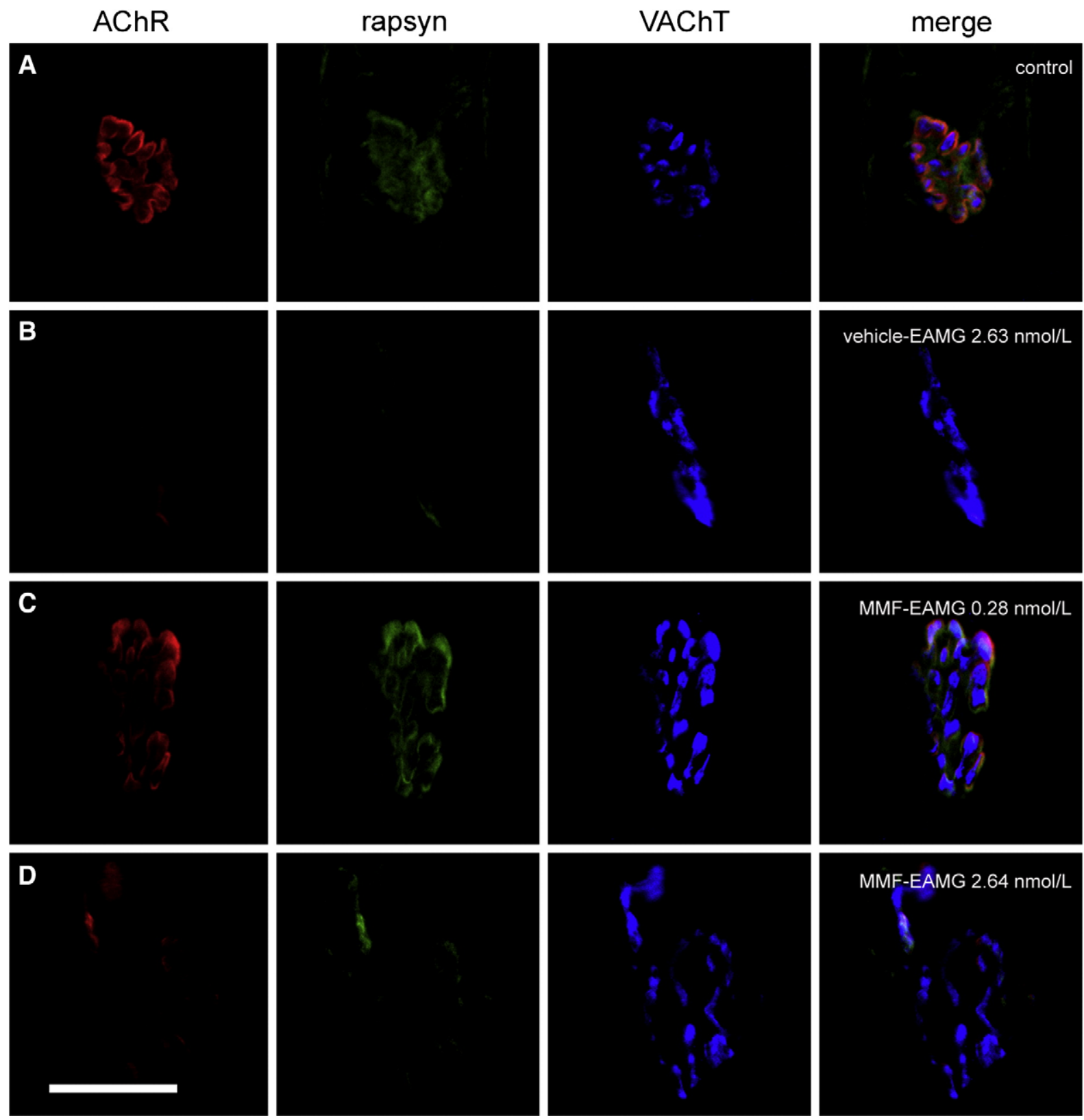

Fig. 4. Cryosections of left tibialis anterior muscles, 8 weeks after immunization, triple stained with Alexa 594-conjugated $\alpha$-bungarotoxin (red), mouse anti-rapsyn $\mathrm{mAb} 1234$ (green) and rabbit anti-VAChT (blue) in the neuromuscular junctions of a representative control, vehicle-treated EAMG animal and two EAMG rats treated for 3 weeks with MMF; merge on the right. Panels correspond to $400 \times$ magnification, bar $=25 \mu \mathrm{m}$. Destruction of motor endplates was dependent on the concentration of anti-rat AChR antibody titers. In all control rats, AChR, rapsyn and VAChT were co-localized at the endplate (A). In contrast, all vehicle-treated chronic EAMG rats showed reduced staining of postsynaptic AChR and rapsyn, but not of presynaptic VAChT (B). In MMF-treated EAMG animals however, normal levels of AChR, rapsyn and VAChT were observed in rats with low antibody titers (C), whereas strongly reduced staining patterns of postsynaptic AChR and rapsyn were observed with high antibody titers (D).

azathioprine exerts its immunosuppressive effect on both the de novo pathway of purine synthesis and the hypoxantine guanine phosphoribosyl transferase salvage pathway; thereby causing not only inhibition of B- and T-lymphocytes proliferation but also inhibition of purine synthesis in other tissues leading among other side effects to bone marrow suppression (Derijks et al., 2004; Gunnarsdottir and Elfarra, 1999). On the other hand, cyclosporine only inactivates T-helper lymphocytes by blockade of interleukin-2 signaling and therefore does not interfere directly with B-lymphocyte proliferation (Hagberg et al., 1988). Since MG is a T-cell dependent and antibodymediated disease, MMF is not only thought to be a safe, but also a potent immunomodulator for MG (Allison and Eugui, 2000).

In the present study we have demonstrated the beneficial effects of MMF on rats immunized with $t \mathrm{AChR}$, as MMF to our knowledge has not yet been tested in an animal model of MG. MMF did not only act as a potent and safe immunosuppressive drug but also clearly improved neuromuscular transmission in EAMG animals. This experimental disease has previously been shown to be an appropriate model for human MG by different 
criteria such as cellular and humoral immunological responses, decrementing responses of muscle action potentials in electromyographic examinations, as well as transitory improvement by anti-cholinesterase drugs (Lennon et al., 1975).

To test whether MMF could prevent the onset of MG in rats, MMF was administered orally for 3 or 8 weeks, starting from the day of immunization. A dose of $30 \mathrm{mg} / \mathrm{kg} /$ day was chosen as this dose has already been proven to be efficient in other experimental diseases (Gibson and Hayden, 2007; Tran et al., 2001; Zandman-Goddard and Shoenfeld, 2005). Moreover, MG patients daily receive an oral dose of 1.0-2.0 g, which is in the range of the body weight related dose of MMF used in the present study (Schneider-Gold et al., 2006). The results showed that MMF was able to suppress anti-rat AChR antibody titers to values to $0.9 \pm 1.3 \mathrm{nmol} / \mathrm{L} 8$ weeks after immunization in both experimental regimes. The immunosuppressive properties of MMF in the present study were moreover consistent with findings in $\mathrm{MG}$ case reports where antibody titers could already be reduced up to $50 \%$ after one month of MMF treatment (Meriggioli et al., 2003b; Schneider et al., 2001).

Compared to other immunosuppressive agents, the improvement in tolerability and safety profile of MMF is has already been established clearly in several small studies. Indeed, while most therapeutic immunosuppressive drugs are known to exert severe nephrotoxic and hepatotoxic side effects, clear adverse effects of MMF treatment have been shown to be absent in most MG patients (Garcia-Carrasco et al., 2007). Although we did not rigorously test for side effects of MMF in our experimental model, none of the MMF-treated animals in this study developed overt side effects such as fever, diarrhea, dehydration, breathing difficulties or immunosuppression related infections. Furthermore, MMF seemed to be highly efficient in preventing clinical MG symptoms in $95 \%$ of immunized animals. Single case reports and several small clinical studies concerning MMF treatment in MG confirm the abovementioned observations as most patients were able to reach pharmacological remission, and improved manual muscle testing and quantitative MG score after several months of treatment (Caponnetto et al., 2001; Chaudhry et al., 2001; Ciafaloni et al., 2001; Hauser et al., 1998; Lim et al., 2007; Meriggioli et al., 2003a; Meriggioli et al., 2003b; Mowzoon et al., 2001; Prakash et al., 2007; Schneider et al., 2001). In contrast, two recently completed large phase 3 prospective, multicenter, placebo-controlled trials showed little or no effect of MMF when it was used as an adjunctive treatment to corticosteroids. Indeed, no improvement was observed in baseline Quantitative Myasthenia Gravis score for disease severity and in the ability to decrease the dose of corticosteroids and cholinesterase inhibitors, as AChR antibody titers decreased equally in both groups by about $50 \%$. However, the obtained results are likely due to a higher than expected response to prednisone alone (Hampton, 2007).

To study the effects of MMF-induced low anti-rat AChR antibody titers on clinical characteristics in more detail, EMG measurements of tibialis anterior muscles were performed during continuous curare infusions. The results indicated a significant improvement in neuromuscular transmission after
MMF administration to EAMG rats. Moreover, no significant differences could be observed between MMF-treated EAMG animals and sham-immunized control rats, indicating that the immunosuppressant action of MMF was indeed highly efficient in improving neuromuscular transmission, which is in accordance with the clinical EAMG score discussed earlier. Patient data fully support these findings as improvements in functional status, manual muscle testing and jitter in single fiber EMG were reported after MMF treatment in MG (Chaudhry et al., 2001; Ciafaloni et al., 2001; Hampton, 2007; Meriggioli et al., 2003a; Meriggioli et al., 2003b; Mowzoon et al., 2001; Prakash et al., 2007). Interestingly, the anti-rat AChR antibody titers observed in the present study were moreover exponentially correlated with curare resistance of neuromuscular transmission. Indeed, values above $1.0 \mathrm{nmol} / \mathrm{L}$ of anti-rat $\mathrm{AChR}$ antibodies seemed to be responsible for provocation of decrementing responses at about $50 \%$ of the normal curare dose. This indicates that in the present setup only $30 \%$ of all MMF-treated EAMG animals would suffer from as severe neuromuscular deficits as occurring in vehicle-treated EAMG rats, since short-term administration of MMF was able to suppress anti-rat $\mathrm{AChR}$ antibody titers below $1.0 \mathrm{nmol} / \mathrm{L}$ in $70 \%$ of immunized rats (Fig. 1B). These findings suggest the possibility that very low, or even undetectable anti-AChR antibody levels can cause the neuromuscular junction transmission defect in MG patients. At the basis of a high susceptibility of some patients to low levels of auto-antibodies might be genetic differences in the expression levels of postsynaptic proteins. As a case in point, it has been shown that rapsyn, utrophin and s-laminin expression determine disease severity in rats susceptible to EAMG (Hoedemaekers et al., 1998; Losen et al., 2005; Martinez-Martinez et al., 2007).

In relation to neuromuscular transmission, tibialis anterior AChR concentrations were also determined. The results were rather surprising as AChR concentrations of both vehicletreated and MMF-treated EAMG animals were equally decreased by about $45 \%$ when compared to sham-immunized controls, whereas anti-rat AChR antibody titers of MMF-treated EAMG rats were 10 times lower in comparison with vehicletreated EAMG animals. On the other hand, correlation analysis revealed that the anti-rat $\mathrm{AChR}$ antibody titer is an important determinant in breaking down the AChRs of the muscle. Indeed, a similar relation was observed between antibody titers and muscle AChR concentration when compared to the previously discussed correlation between anti-rat $\mathrm{AChR}$ antibodies and neuromuscular transmission. Severe AChR breakdown up to $50 \%$ of normal values already seemed to occur with an antirat AChR antibody titer of about $1.0 \mathrm{nmol} / \mathrm{L}$. Immunohistochemical analyses of endplate regions fully confirmed these findings. Vehicle-treated EAMG rats seemed to have an overall breakdown of neuromuscular junction AChRs, while endplate AChRs of MMF-treated EAMG animals were not equally destructed. In particular, it seemed that overall endplate AChRs were intact with extremely low antibody titers while AChR breakdown was observed in several neuromuscular junctions of MMF-treated animals with higher anti-rat AChR antibody titers. It is possible that the membrane AChR loss measured in 
muscle extracts is in part due to loss of unclustered and more susceptible extrasynaptic AChRs. The electromyographical measurements during challenge with curare are however certainly suggestive of AChR protection at the neuromuscular junction as a result of MMF treatment.

In summary, these data show that short-term administration of MMF is able to act as a highly immunosuppressive drug in an animal model of MG and consequently improves clinical characteristics by enhancing neuromuscular transmission. Moreover, these data strengthen the case for using MMF as a treatment of human MG, although further large clinical trials are required to determine the exact use of MMF as a sole therapeutic or as an adjunctive treatment to other immunosuppressive drugs.

\section{Acknowledgements}

The authors sincerely thank Aspreva Pharmaceuticals, Palo Alto, United States for their financial support and for providing the MMF.

\section{References}

Allison, A.C., Eugui, E.M., 2000. Mycophenolate mofetil and its mechanisms of action. Immunopharmacology 47, 85-118.

Blaheta, R.A., Leckel, K., Wittig, B., Zenker, D., Oppermann, E., Harder, S., Scholz, M., Weber, S., Schuldes, H., Encke, A., Markus, B.H., 1998. Inhibition of endothelial receptor expression and of T-cell ligand activity by mycophenolate mofetil. Transpl. Immunol. 6, 251-259.

Caponnetto, C., Rossi, E., Primavera, A., 2001. Mycophenolate mofetil: a new immunosuppressive approach. Successful treatment in a case of myasthenia gravis associated with incomplete lupus erythematosus syndrome and hepatitis C virus infection. Eur. Neurol. 46, 53-54.

Chaudhry, V., Cornblath, D.R., Griffin, J.W., O'Brien, R., Drachman, D.B., 2001. Mycophenolate mofetil: a safe and promising immunosuppressant in neuromuscular diseases. Neurology 56, 94-96.

Ciafaloni, E., 2005. Mycophenolate mofetil and myasthenia gravis. Lupus 14 (Suppl 1), s46-49.

Ciafaloni, E., Massey, J.M., Tucker-Lipscomb, B., Sanders, D.B., 2001. Mycophenolate mofetil for myasthenia gravis: an open-label pilot study. Neurology 56, 97-99.

Cohn, R.G., Mirkovich, A., Dunlap, B., Burton, P., Chiu, S.H., Eugui, E., Caulfield, J.P., 1999. Mycophenolic acid increases apoptosis, lysosomes and lipid droplets in human lymphoid and monocytic cell lines. Transplantation 68, 411-418.

De Baets, M., Stassen, M.H., 2002. The role of antibodies in myasthenia gravis. Ann. N.Y. Acad. Sci. 202, 5-11.

De Baets, M., Stassen, M., Losen, M., Zhang, X., Machiels, B., 2003. Immunoregulation in experimental autoimmune myasthenia gravis-about $\mathrm{T}$ cells, antibodies, and endplates. Ann. N.Y. Acad. Sci. 998, 308-317.

Derijks, L.J., Gilissen, L.P., Engels, L.G., Bos, L.P., Bus, P.J., Lohman, J.J., Curvers, W.L., Van Deventer, S.J., Hommes, D.W., Hooymans, P.M., 2004. Pharmacokinetics of 6-mercaptopurine in patients with inflammatory bowel disease: implications for therapy. Ther. Drug. Monit. 26, 311-318.

Drachman, D.B., 1994. Myasthenia gravis. N. Engl. J. Med. 330, 1797-1810.

Durez, P., Appelboom, T., Pira, C., Stordeur, P., Vray, B., Goldman, M., 1999. Antiinflammatory properties of mycophenolate mofetil in murine endotoxemia: inhibition of TNF-alpha and upregulation of IL-10 release. Int. J. Immunopharmacol. 21, 581-587.

Enk, A.H., Knop, J., 1997. Treatment of pemphigus vulgaris with mycophenolate mofetil. Lancet 350, 494.

Eugui, E.M., Mirkovich, A., Allison, A.C., 1991. Lymphocyte-selective antiproliferative and immunosuppressive activity of mycophenolic acid and its morpholinoethyl ester (RS-61443) in rodents. Transplant. Proc. 23, 15-18.
European Mycophenolate Mofetil Cooperative Study Group, 1995. Placebocontrolled study of mycophenolate mofetil combined with cyclosporin and corticosteroids for prevention of acute rejection. Lancet 345, $1321-1325$.

Gajdos, P., Chevret, S., Toyka, K., 2006. Intravenous immunoglobulin for myasthenia gravis. Cochrane. Database. Syst. Rev. CD002277.

Garcia-Carrasco, M., Escarcega, R.O., Fuentes-Alexandro, S., Riebeling, C., Cervera, R., 2007. Therapeutic options in autoimmune myasthenia gravis. Autoimmun. Rev. 6, 373-378.

Gibson, W.T., Hayden, M.R., 2007. Mycophenolate mofetil and atherosclerosis: results of animal and human studies. Ann. N. Y. Acad. Sci. 1110, 209-221.

Ginzler, E.M., Dooley, M.A., Aranow, C., Kim, M.Y., Buyon, J., Merrill, J.T., Petri, M., Gilkeson, G.S., Wallace, D.J., Weisman, M.H., Appel, G.B., 2005. Mycophenolate mofetil or intravenous cyclophosphamide for lupus nephritis. N. Engl. J. Med. 353, 2219-2228.

Goldblum, R., 1993. Therapy of rheumatoid arthritis with mycophenolate mofetil. Clin. Exp. Rheumatol. 11 (Suppl 8), S117-119.

Gronseth, G.S., Barohn, R.J., 2000. Practice parameter: thymectomy for autoimmune myasthenia gravis (an evidence-based review): report of the Quality Standards Subcommittee of the American Academy of Neurology. Neurology 55, 7-15.

Gunnarsdottir, S., Elfarra, A.A., 1999. Glutathione-dependent metabolism of cis-3-(9H-purin-6-ylthio)acrylic acid to yield the chemotherapeutic drug 6mercaptopurine: evidence for two distinct mechanisms in rats. J. Pharmacol. Exp. Ther. 290, 950-957.

Hagberg, R.C., Hoyt, E.G., Billingham, M.E., Sibley, R.K., Starnes, V.A., Baldwin, J.C., 1988. Comparison of cyclosporin A and G with and without azathioprine regarding immunosuppressive efficacy, toxicity, and pharmacokinetics in Lewis rats. J. Heart. Transplant. 7, 359-369.

Hampton, T., 2007. Trials assess myasthenia gravis therapies. Jama 298, 29-30.

Hauser, R.A., Malek, A.R., Rosen, R., 1998. Successful treatment of a patient with severe refractory myasthenia gravis using mycophenolate mofetil. Neurology 51, 912-913.

Hoch, W., McConville, J., Helms, S., Newsom-Davis, J., Melms, A., Vincent, A., 2001. Auto-antibodies to the receptor tyrosine kinase MuSK in patients with myasthenia gravis without acetylcholine receptor antibodies. Nat. Med. 7, 365-368

Hoedemaekers, A., Graus, Y., van Breda Vriesman, P., de Baets, M., 1997. Ageand sex-related resistance to chronic experimental autoimmune myasthenia gravis (EAMG) in Brown Norway rats. Clin. Exp. Immunol. 107, 189-197.

Hoedemaekers, A., Bessereau, J.L., Graus, Y., Guyon, T., Changeux, J.P., Berrih-Aknin, S., van Breda Vriesman, P., De Baets, M.H., 1998. Role of the target organ in determining susceptibility to experimental autoimmune myasthenia gravis. J. Neuroimmunol. 89, 131-141.

Larkin, G., Lightman, S., 1999. Mycophenolate mofetil. A useful immunosuppressive in inflammatory eye disease. Ophthalmology 106, 370-374.

Lehmann, H.C., Hartung, H.P., Hetzel, G.R., Stuve, O., Kieseier, B.C., 2006. Plasma exchange in neuroimmunological disorders: part 2. Treatment of neuromuscular disorders. Arch. Neurol. 63, 1066-1071.

Lennon, V.A., Lindstrom, J.M., Seybold, M.E., 1975. Experimental autoimmune myasthenia: a model of myasthenia gravis in rats and guinea pigs. J. Exp. Med. 141, 1365-1375.

Lim, A.K., Donnan, G., Chambers, B., Ierino, F.L., 2007. Mycophenolate mofetil substitution for cyclosporine-dependent myasthenia gravis and nephrotoxicity. Intern. Med. J. 37, 55-59.

Lindstrom, J.M., Seybold, M.E., Lennon, V.A., Whittingham, S., Duane, D.D., 1976. Antibody to acetylcholine receptor in myasthenia gravis. Prevalence, clinical correlates, and diagnostic value. Neurology 26, 1054-1059.

Losen, M., Stassen, M.H., Martinez-Martinez, P., Machiels, B.M., Duimel, H., Frederik, P., Veldman, H., Wokke, J.H., Spaans, F., Vincent, A., De Baets, M.H., 2005. Increased expression of rapsyn in muscles prevents acetylcholine receptor loss in experimental autoimmune myasthenia gravis. Brain 128, 2327-2337.

Losen, M., Martínez-Martínez, P., Phernambucq, M., Schuurman, J., Parren, P.W., De Baets, M.H., 2008. Treatment of myasthenia gravis by preventing acetylcholine receptor modulation. Ann. N.Y. Acad. Sci. 1132, 174-179.

Martinez-Martinez, P., Losen, M., Duimel, H., Frederik, P., Spaans, F., Molenaar, P., Vincent, A., De Baets, M.H., 2007. Overexpression of rapsyn 
in rat muscle increases acetylcholine receptor levels in chronic experimental autoimmune myasthenia gravis. Am. J. Pathol. 170, 644-657.

Meriggioli, M.N., Ciafaloni, E., Al-Hayk, K.A., Rowin, J., Tucker-Lipscomb, B., Massey, J.M., Sanders, D.B., 2003a. Mycophenolate mofetil for myasthenia gravis: an analysis of efficacy, safety, and tolerability. Neurology 61, 1438-1440.

Meriggioli, M.N., Rowin, J., Richman, J.G., Leurgans, S., 2003b. Mycophenolate mofetil for myasthenia gravis: a double-blind, placebo-controlled pilot study. Ann. N. Y. Acad. Sci. 998, 494-499.

Mowzoon, N., Sussman, A., Bradley, W.G., 2001. Mycophenolate (CellCept) treatment of myasthenia gravis, chronic inflammatory polyneuropathy and inclusion body myositis. J. Neurol. Sci. 185, 119-122.

Neurath, M.F., Wanitschke, R., Peters, M., Krummenauer, F., Meyer zum Buschenfelde, K.H., Schlaak, J.F., 1999. Randomised trial of mycophenolate mofetil versus azathioprine for treatment of chronic active Crohn's disease. Gut 44, 625-628.

Nowack, R., Gobel, U., Klooker, P., Hergesell, O., Andrassy, K., van der Woude, F.J., 1999. Mycophenolate mofetil for maintenance therapy of Wegener's granulomatosis and microscopic polyangiitis: a pilot study in 11 patients with renal involvement. J. Am. Soc. Nephrol. 10, 1965-1971.

Palace, J., Newsom-Davis, J., Lecky, B., 1998. A randomized double-blind trial of prednisolone alone or with azathioprine in myasthenia gravis. Myasthenia Gravis Study Group. Neurology 50, 1778-1783.

Patel, A.A., Swerlick, R.A., McCall, C.O., 2006. Azathioprine in dermatology: the past, the present, and the future. J. Am. Acad. Dermatol. 55, 369-389.

Prakash, K.M., Ratnagopal, P., Puvanendran, K., Lo, Y.L., 2007. Mycophenolate mofetil — as an adjunctive immunosuppressive therapy in refractory myasthenia gravis: the Singapore experience. J. Clin. Neurosci. 14, $278-281$.

Richman, D.P., Agius, M.A., 2003. Treatment of autoimmune myasthenia gravis. Neurology 61, 1652-1661.

Sanders, D.B., Hart, I.K., Mantegazza, R., Shukla, S.S., Siddiqi, Z.A., De Baets, M.H., Melms, A., Nicolle, M.W., Solomons, N., Richman, D.P., 2008. An international, phase III, randomized trial of mycophenolate mofetil in myasthenia gravis. Neurology. doi:10.1212/01.wnl.0000312374.95186.cc.

Schneider-Gold, C., Hartung, H.P., Gold, R., 2006. Mycophenolate mofetil and tacrolimus: new therapeutic options in neuroimmunological diseases. Muscle. Nerve. 34, 284-291.

Schneider, C., Gold, R., Reiners, K., Toyka, K.V., 2001. Mycophenolate mofetil in the therapy of severe myasthenia gravis. Eur. Neurol. 46, 79-82.

Seybold, M.E., Lambert, E.H., Lennon, V.A., Lindstrom, J.M., 1976. Experimental autoimmune myasthenia: clinical, neurophysiologic, and pharmacologic aspects. Ann. N. Y. Acad. Sci. 274, 275-282.

Sieb, J.P., 2005. Myasthenia gravis: emerging new therapy options. Curr. Opin. Pharmacol. 5, 303-307.

The Muscle Study Group, 2008. A trial of mycophenolate mofetil with prednisone as initial immunotherapy in myasthenia gravis. Neurology. doi:10.1212/01.wnl.0000312374.95186.cc.

Tran, G.T., Carter, N., Hodgkinson, S.J., 2001. Mycophenolate mofetil treatment accelerates recovery from experimental allergic encephalomyelitis. Int. Immunopharmacol. 1, 1709-1723.

Zandman-Goddard, G., Shoenfeld, Y., 2005. Mycophenolate mofetil in anima models of autoimmune disease. Lupus 14 (Suppl 1), s12-16. 